Meta

Journal des traducteurs

Translators' Journal

\title{
Terminologie ou terminologies? Spécialité linguistique ou domaine interdisciplinaire?
}

\section{Teresa Cabré}

Volume 36, numéro 1, mars 1991

La terminologie dans le monde : orientations et recherches

URI : https://id.erudit.org/iderudit/002184ar

Aller au sommaire du numéro

Éditeur(s)

Les Presses de l'Université de Montréal

ISSN

0026-0452 (imprimé)

Découvrir la revue

Citer cet article

Cabré, M. T. (1991). Terminologie ou terminologies? Spécialité linguistique ou domaine interdisciplinaire? Meta, 36(1), 55-63. d'utilisation que vous pouvez consulter en ligne. 


\title{
TERMINOLOGIE OU TERMINOLOGIES? SPÉCIALITE LINGUISTIQUE OU DOMAINE INTERDISCIPLINAIRE?
}

\author{
M. Teresa CABrÉ \\ Université de Barcelone, Barcelone, Espagne
}

\section{INTRODUCTION}

Cet article est une réflexion sur la délimitation de la terminologie, plutôt qu'une recherche sur son but ou son champ de travail. Le but fondamental qu'il poursuit est de s'interroger sur :

1) la diversification actuelle de la notion de terminologie,

2) sa place parmi les sciences et les techniques,

3) la nécessité de la délimiter pour mieux définir la/les méthode(s) de travail terminographique et l'organisation même du travail de recherche.

Ce sujet n'est pas pour nous un thème banal; bien au contraire, il a été un motif de réflexion systématique depuis 1986, lors de notre direction du Centre de Terminologie de la Catalogne. Nous avons cru percevoir qu'il y avait quelque chose dans l'organisation du travail de recherche - inspiré fondamentalement de l'organisation québécoise - qui présentait des difficultés. Cela nous a fait remettre en question quelques éléments du processus de travail et de l'organisation des tâches. L'expérience vécue à la Commission de Normalisation Terminologique (Conseil Superviseur de la Terminologie) nous a aidée à systématiser notre opinion sur ce sujet. Enfin, la lecture de publications partant de conceptions différentes nous a permis d'acquérir une vision diversifiée des faits que nous allons tenter d'analyser maintenant.

\section{LA NORMALISATION TECHNIQUE ET SCIENTIFIQUE}

Aujourd'hui il n'y a personne, parmi les spécialistes en terminologie, qui ne puisse ignorer que le concept de «normalisation» est né chez les techniciens et les scientifiques du premier quart du siècle :

La nécessité de la normalisation terminologique est d'abord apparue dans les années 1930 1940 parmi les spécialistes, qu'ils soient des individus (Ernest Dresen en URSS, E. Wüster en Autriche) ou des groupes (Commission Électrotechnique Internationale, Commission de la réforme de la nomenclature de la chimie organique). C'est à eux que revient la définition du concept de normalisation terminologique et des mécanismes consultatifs de sa mise en application. De cette réflexion est né en 1937 le Comité de normalisation de l'ISA d'où sortira plusieurs années plus tard le Comité technique 37 de l'ISO. (Auger 1980: 146)

La normalisation de la terminologie des domaines de spécialité naît de la nécessité pour les spécialistes de dénommer les nouveaux produits et concepts dans leurs domaines, résultat du rapide développement de la science et de la technique, et de l'obligation d'établir une communication univoque dans ces domaines:

The rapid increase in knowledge and in the production of technical goods made it necessary for scholars, scientists, engineers and craftsmen to deal with language, i.e. to name and 
define newly created objects and new scientific phenomena. Systems of terms were developped in biology by Linné in 1735 and in 1782 by Morveau for chemistry.(...) In the 19 th century, international cooperation in science necessitated the development of unified rules for the formation of terms. This started at the international congresses of botanists in 1867, of zoologists in 1889 and of chemists in 1892. (...) In the 20th century the rapid growth of technology made it necessary for engineers to direct their attention to linguistic problems in technology. The most eminent researchers are: Wüster (1898-1977) in Austria (...) and Lotte (1889-1950) in the Soviet Union. (Felber 1985)

Le but de la normalisation, conçue en ce sens, est, pour les spécialistes, le transfert de la connaissance. Donc, la terminologie, conçue comme discipline et comme pratique:

a) doit permettre une interconnexion entre les spécialistes du même domaine, qu'ils parlent ou non la même langue,

b) doit se centrer fondamentalement sur le spécialiste, parce que c'est lui qui en a besoin,

c) doit permettre le transfert des connaissances sans ambiguité, plutôt que l'expression dans la langue maternelle.

Par conséquent, normaliser, dans cette conception de la terminologie, signifie standardiser pour mieux se comprendre; donc, appauvrir la langue en faveur de la communication.

La terminologie est ici une affaire de spécialistes, et il leur revient de faire (ou de gérer) la recherche terminologique et de décider par consensus les dénominations standardisées.

Les domaines de leur recherche sont ceux des disciplines scientifico-techniques.

\section{LA TERMINOLOGIE, OUTIL DE NORMALISATION LINGUISTIQUE}

Deux événements de conséquences apparemment contradictoires sont le résultat des changements sociaux et politiques survenus après la Deuxième Guerre mondiale :

1. l'apparition d'institutions, entreprises et marchés internationaux (et donc plurilingues), d'où la nécessité d'agir avec diverses langues ensemble et d'assurer leurs équivalences terminologiques.

2. La puissance des nationalismes identificatifs; d'où la nécessité de chercher des alternatives de dénomination dans la propre langue, valables pour les nouvelles réalités créées ailleurs.

L'intervention des gouvernements en affaires linguistiques a demandé l'élaboration de plans de politique linguistique destinés à protéger le statut d'une ou de plusieurs langues dans un territoire administratif. Ainsi, ont fait leur apparition les plans de protection, non seulement des langues minoritaires en conflit ou minorisées dans leur territoire, mais aussi des langues puissantes historiquement, sans conflit dans leur territoire administratif, mais aujourd'hui menacées par la puissance monopolisatrice de l'anglais.

Les politiques linguistiques d'intervention officielle ont inclus la terminologie dans leur programme, déjà qu'une langue se réaffirme si elle est capable de s'adapter aux nouveautés. Pour ccla, plusieurs pays avec les langues en situation de conflit ont créé des centres de terminologie dépendants d'organismes officiels pour mener les travaux terminologiques, régler la néologie et garantir l'implantation des nouveaux termes dans les milieux concernés.

Dans ce cadre d'agissement social et politique, on conçoit la terminologie comme une des multiples manifestations de la vitalité d'une langue. Les travaux terminologiques n'ont pas pour objectif premier d'assurer l'univocité du transfert de connaissances entre 
spécialistes, mais plutôt de témoigner de la capacité de la langue à répondre au défi de la modernité sans intermédiaires.

La conception de la terminologie, tant comme discipline que comme pratique, change par rapport à la notion telle que perçue par les spécialistes. Dans le cadre de cette nouvelle approche, la terminologie:

a) doit permettre une interconnexion entre des spécialistes ayant la même langue;

b) doit être axée sur le sujet parlant, et, pourtant, vécue comme une affaire prioritairement linguistique, plutôt que comme une affaire scientifique et technique;

c) doit être un témoin de la vitalité et de la modernité d'une langue, qui permette le transfert des connaissances.

Nous soulignons que la notion de normalisation a changé un peu par rapport à celle que nous avons présentée auparavant. Normaliser signifie ici conquérir des domaines spécialisés pour la langue d'expression habituelle.

Si la terminologie est considérée comme une affaire linguistique, c'est donc aux linguistes d'en gérer la recherche. Dans cette approche, les sujets de recherche se diversifient, déjà qu'ils concernent tous les sujets parlants, et non pas seulement les spécialistes. Néanmoins, il est difficile d'établir ce qu'on appelle domaines de spécialité:

Special languages or more precisely subject languages, are usually thought of as the means of expression of highly qualified subject specialists like engineers, physicians, lawyers, etc. and are often derogatively referred to as ujargon». The fact that humbler occupations like nursing, book-keeping and cooking and even hobbies also involve special areas of human interest and therefore also require and indeed have their own special language is much less often acknowledged. Since practically every human activity can be attributed to one subject or another, all language could be split into so many subject languages and the word "special" would be superfluous. (Sager 1980)

$\mathrm{Si}$ un processus de normalisation linguistique réglé par une politique de la langue doit combler tous les domaines de communication où la langue est employée, un plan de recherche terminologique doit concerner tous les domaines de communication où la terminologie a une place, tous les domaines spécialisés au sens le plus large possible. En ce sens, il doit concerner:

a) le lexique commun,

b) les lexiques spécialisés de premier degré ou vocabulaires de tronc commun (lexiques-charnière ou banalisés),

c) les lexiques proprement spécialisés ou terminologies scientifico-techniques, en adoptant deux points de vue

1. le point de vue du producteur,

2. le point de vue du consommateur.

Dans la première conception de la terminologie comme étant une affaire de spécialistes, seul le domaine 1 est un objet pertinent de recherche; dans la seconde, tous les domaines le sont.

\section{LA CONFUSION TERMINOLOGIQUE}

Quand on parle de terminologie, une certaine confusion est le résultat:

a) d'un manque de différenciation entre les deux approches que nous venons de présenter,

b) d'une conception excessivement uniforme de la méthodologie de recherche,

c) d'une application trop homogène de cette méthodologie dans tous les domaines de spécialité,

d) d'un manque de distinction entre les différents buts à atteindre. 
Cette confusion se retrouve à trois niveaux :

a) dans la discipline,

b) dans la méthodologie,

c) dans les aspects de l'organisation du travail terminologique.

Par conséquent, et pour mieux agir, il serait nécessaire de clarifier des questions portant sur:

- le champ de travail de la terminologie proprement dite. Faut-il faire une différence entre deux sortes de terminologie? Est-ce qu'il serait mieux d'employer un mot différent pour désigner ces différences?

- les agents de la recherche. Est-ce que la distinction entre les divers types de «terminologie» peut conditionner les chercheurs?

- les buts de la recherche. Est-ce que le but détermine le type de recherche?

- la raison pour laquelle se fait une recherche systématique? Où doit-elle conduire?

— les «autorités» terminologiques. Qui peut décider en terminologie? Sur quelle sorte d'autorité faut-il compter pour standardiser les termes? tels que :

Une fois ces questions éclaircies, nous pourrons discuter de points fondamentaux

a) la délimitation des domaines de recherche,

b) le statut de la terminologie parmi les sciences et son rapport à la linguistique,

c) le processus de travail en terminographie,

d) la provenance scientifique du terminologue et la diversification de la formation,

e) la notion «d'autorité valable» en normalisation,

f) la présentation des lexiques et des dictionnaires,

g) les diverses stratégies d'implantation/diffusion de la terminologie nouvelle.

\section{LA DIVERSIFICATION NÉCESSAIRE}

Aujourd'hui, parler de terminologie signifie se situer dans une approche de recherche et donc de conception théorique et méthodologique déterminées. On ne peut pas travailler en terminologie sans préciser que le point de départ est $\mathrm{x}$, le but qu'on poursuit est y, et la cause, $z$.

À notre avis, la diversification de la terminologie concerne quatre éléments fondamentaux : l'objet de la recherche, les agents de la recherche, les buts à atteindre et les agents de la normalisation.

\section{L'OBJET DE LA RECHERCHE}

Il faut faire la distinction entre trois couches de lexique du point de vue de la spécialisation ${ }^{1}$ :

a) le lexique général,

b) le lexique spécialisé ou lexique-charnière,

c) la terminologie proprement dite.

Le lexique général est utilisé pour tout type de communication, même spécialisée; il apparaît mêlé avec les termes spécialisés.

Dans tous les langages de spécialité, l'analyse des textes montre un pourcentage important de lexique commun, et un pourcentage variable de lexique-charnière et de terminologie. La variabilité de la quantité de terminologie est conditionnée par le degré d'abstraction du texte. Le lexique-charnière est utilisé dans les textes spécialisés de large diffusion (dans le discours des médias, de l'enseignement, de présentation d'un produit, etc.), destinés au grand public ou à un public peu informé sur le sujet concerné. 
Par contre, la terminologie proprement dite représente le bloc restreint du vocabulaire utilisé par des spécialistes communiquant entre eux.

Si on accepte notre distinction, il s'ensuit que la thématique du domaine, à côté du degré d'abstraction du discours, conditionne le pourcentage de lexique commun et spécialisé. Cela explique que tous les domaines montrent diverses couches de lexiques (des moins aux plus spécialisées) qui sont utilisés en priorité par les sujets parlants en fonction du caractère plus ou moins restreint de la communication. En même temps, à mesure que la communication dans un domaine progresse, le pourcentage de lexique spécialisé augmente.

La biochimie, la robotique, la production mécanique ont leur vocabulaire spécialisé ou terminologique distribué en diverses couches successives de spécialisation. Tous trois ont une quantité considérable de lexique restreint spécifique et particulier connu par les spécialistes en la matière. Un locuteur parlant de biochimie, de robotique ou de production mécanique utilisera ce vocabulaire spécialisé, en le combinant avec des mots du lexique commun ou en ayant recours à la paraphrase ou à des explications incidentes selon le degré de spécialisation de l'interlocuteur. Pendant la communication il réduira progressivement le lexique de base en faveur d'une augmentation du lexique spécialisé, parce que l'interlocuteur est «incorporé» progressivement à la «spécialité» discursive. La progression de la situation discursive nuance donc le pourcentage de «spécialité».

D'autres domaines comme les sports, les activités se rapportant au loisir, à la boulangerie ou à la mode, ont aussi leur vocabulaire restreint; mais, vu qu'il sert surtout pour communiquer avec le grand public, ce vocabulaire est moins important que celui des cas antérieurs.

À la question, donc, de «qu'est-ce qui doit s'appeler terminologie?» il faudrait répondre qu'il est fondamental de faire une distinction entre les lexiques spécialisés de grande diffusion, et la terminologie scientifique et technique spécifique des diverses disciplines qui sont l'objet de recherches théoriques, de recherches appliquées, d'applications, de productions et de commercialisations, et d'enseignement à tous les niveaux. Si on ne fait pas cette distinction, on court le risque de vider de son sens le mot «terminologie».

Since practically every human activity can be atributed to one subject or another, all language could be split into so many subjectlanguages and the word «special» would be superfluous. (Sager 1980)

\section{LES AGENTS TERMINOLOGIQUES}

On ne peut pas oublier que les vrais agents de la terminologie sont ses usagers. Ce sont les usagers qui perçoivent la nécessité dénominative, qui se heurtent au terme emprunté, qui se trouvent devant la lacune dénominative.

Déterminer donc les usagers de la terminologie conduit logiquement à sélectionner les agents les mieux placés pour en faire la recherche.

Sur ce point, il faut distinguer entre les deux types établis auparavant: les lexiques de spécialité (LS) et la terminologie scientifico-technique (TST).

En ce qui concerne la terminologie scientifique et technique (TST), ce serait les scientifiques et les techniciens les seuls connaisseurs du domaine - sauf exception et donc les meilleurs chercheurs de dénominations de concepts spécifiques.

Les LS, vu qu'ils sont connus par une bonne partie de la population, ne demanderaient pas une spécialisation thématique des chercheurs aussi stricte que dans le cas de la TSC; ils n'exigeraient qu'une bonne formation méthodologique de recherche en terminologie pour que les résultats obtenus soient de bonne qualité. 
Mais les spécialistes en tant que spécialistes de la terminologie n'ont pas la préparation adéquate pour faire de la recherche terminologique. Donc, il faut les former en méthodologie. terrains:

La recherche terminologique exige une information/formation sur plusieurs

a) la situation et l'organisation du milieu spécifique,

b) le contenu du domaine,

c) les bases conceptuelles de la terminologie,

d) le processus de travail,

e) les normes de présentation du travail,

f) les recours linguistiques et les voies de transmission des propositions nouvelles pour implanter et diffuser la terminologie.

Il est évident que les points a et $b$ ne sont connus que par les spécialistes et il leur incombe de faire (ou de gérer) les travaux se rapportant à la description et à l'exploration du milieu, la recherche des sources, la fixation du corpus de travail, la structuration conceptuelle du domaine, la reconnaissance des termes pertinents, la validation des équivalents et des définitions; en bref, toutes les tâches concernant le milieu, les signifiés, les contenus. Et il leur revient aussi de proposer les dénominations pour combler une lacune formelle ou pour remplacer une dénomination inadéquate du point de vue $d u$ spécialiste.

Alors, est-ce que le linguiste, en tant que linguiste, ne joue pas un rôle spécial dans la recherche? À notre avis, pas tellement important en ce qui concerne la terminologie ST; au moins pas si important que le scientifique ou que le technicien. C'est une question pragmatique qui part de la considération que tel type de spécialiste est le mieux placé par sa formation de base pour diriger (gérer, pas nécessairement réaliser) la recherche.

Évidemment, en TST c'est aux spécialistes, à notre avis, qu'il appartient de mener l'initiative de la recherche, car ils sont les seuls légitimés pour la faire. Ils sont les seuls qui connaissent le milieu, le contenu du domaine et la terminologie, bien ou mal utilisée. Par conséquent, ils sont les agents les mieux placés pour réaliser les tâches décrites auparavant. Pour cela, les spécialistes ont besoin d'une formation spécifique en méthodologie de la recherche proprement terminologique qui évite la confusion entre l'activité terminologique et l'activité traductionnelle; qui distingue clairement entre la recherche de termes et l'établissement de dénominations; qui établisse nettement qu'un terme est une unité conceptuelle et formelle, et non pas un segment graphique ou phonique. Cela signifie que le spécialiste, s'il veut mener une recherche, doit être formé comme terminologue.

Mais la pratique terminologique ne se limite pas à la recherche des dénominations, à l'établissement des équivalents, à l'attribution des définitions; elle comporte aussi la présentation des résultats de la recherche sous la forme de texte spécialisé, formellement structuré : les glossaires, les lexiques, les dictionnaires. Cela signifie, par conséquent, qu'il faut compléter le travail avec des tâches spécifiques de présentation que ni le spécialiste ni le linguiste ne connaissent, à moins qu'ils n'aient reçu une formation additionnelle spécifique.

Ce deuxième groupe de tâches qui peuvent être effectuées tant par un spécialiste que par un linguiste doit être l'objet de la formation d'un terminologue.

Si le linguiste est formé spécifiquement comme terminologue dans des aspects comme la terminologie générale, la méthodologie de la recherche terminologique et la normalisation terminologique internationale, il peut, évidemment, réaliser ces tâches souvent mieux qu'un spécialiste, car il connaît déjà les éléments de base de la sémantique, de la lexicologie, de la lexicographie générale, etc. 
C'est aussi l'affaire des linguistes de connaître les recours du système de la langue et de donner les possibilités de dénomination pour combler une lacune conceptuelle. Mais c'est l'affaire des spécialistes de favoriser une des solutions proposées en fonction des conditions d'implantation. Évidemment, cela n'arrive que si les spécialistes connaissent bien le milieu, sont au courant des solutions internationales et partagent les critères de politique linguistique et terminologique d'une société spécifique.

De l'argumentation que nous venons de faire, il faut retenir la distinction entre diriger ou mener l'initiative d'une recherche terminologique et réaliser un travail de recherche.

$\grave{A}$ notre avis, l'initiative d'une recherche en terminologie proprement dite doit appartenir aux spécialistes ayant une formation terminologique de base; eux seuls peuvent gérer les tâches relatives au milieu et aux contenus. S'ils ont aussi une formation en méthodologie et en présentation des travaux, ils peuvent sans doute agir en tant que terminologues au sens plein du terme. S'ils ne l'ont pas, il faut qu'ils comptent sur le linguiste formé comme terminologue qui travaillera sous leur direction.

La situation est tout à fait différente pour les recherches des lexiques spécialisés (LS), parce que la connaissance que la majorité de la population dans ce domaine fait que l'initiative des professionnels ne soit pas tellement indispensable et que les linguistes puissent être les vrais agents (directeurs et réalisateurs) du travail, et les «spécialistes» de ces domaines simples informateurs des linguistes.

$\mathrm{Si}$, en outre, on considère que les recherches dans les domaines LS constituent surtout une affaire de normalisation d'une langue, ce sont les linguistes qui sont les plus sensibilisés et les mieux préparés pour mener ces tâches de recherche et d'élaboration des travaux.

En bref, on ne discute pas la nécessité de constituer des équipes mixtes de recherche terminologique, mais il faut distinguer qui a la direction de la recherche dans chaque cas.

\section{JUSTIFICATION ET FINALITÉ DE LA RECHERCHE TERMINOLOGIQUE}

Comme nous l'avons déjà dit, la cause qui stimule la mise en route d'une recherche systématique en terminologie n'est pas toujours la même et les buts qu'on poursuit ne sont pas non plus toujours identiques.

Il est nécessaire de distinguer entre deux finalités de recherche pour éviter la confusion : la normalisation et la description.

Le but le plus souvent poursuivi en terminologie est la standardisation des termes d'un domaine de spécialité, avec deux finalités précises:

a) la normalisation d'une discipline spécialisée (parce que c'est une matière nouvelle, ou envisagée de manière nouvelle, ou c'est un domaine si confus du point de vue conceptuel qu'il a besoin d'une restructuration de son réseau de contenus, etc.);

b) la normalisation de l'usage d'une langue (parce qu'elle possède un certain nombre de lacunes dues à sa situation historique, à des modifications administratives, etc.). La nouveauté de certains domaines provoque un manque de dénominations adéquates ou une incorporation abusive de mots d'emprunt.

Mais les recherches terminologiques peuvent être réalisées aussi avec une intention descriptive, et non nécessairement prescriptive, car il y a beaucoup de domaines où on ne peut pas normaliser la terminologie (les domaines professionnels en voie de disparition), où il est très difficile de la normaliser (plusieurs disciplines sociales et humaines où l'impossibilité d'isoler les concepts provoque une multiplication des points de vue et donc une diversification conceptuelle du même objet). 


\section{LES AUTORITÉS EN STANDARDISATION TERMINOLOGIQUE}

On sait que les agents de la terminologie, ceux qui font des travaux de recherche, ceux qui agissent comme terminologues, ne peuvent pas décider de la forme définitive qu'il faut adopter pour la dénomination d'un concept nouveau et du rejet des formes diverses. C'est seulement l'«autorité» qui peut faire cela. Mais, qu'est-ce que l'«autorité»?

Sur ce point, il faudrait distinguer entre la terminologie dans la perspective de la normalisation d'une discipline, faite par et pour les spécialistes, de la terminologie dans un plan de normalisation d'une langue.

Dans le premier cas, c'est la seule autorité des participants qui agit comme standardisateur. C'est le consensus entre les spécialistes qui fait marcher la normalisation; ils veulent se comprendre sans ambiguité; alors, il faut qu'ils s'accordent sur les formes valables, univoques, standardisées.

Mais la situation n'est pas si simple dans le deuxième cas, parce que chaque société, chaque langue a un statut particulier et précis, qui réclame un traitement spécifique. Et, en plus, la terminologie est vécue commc une affaire plutôt linguistique.

\section{À TITRE DE CONCLUSION}

Comme nous l'avons dit au début, cet article se veut une réflexion indirecte sur trois points: la délimitation et la définition de la terminologie, envisagée tant comme discipline que comme pratique; la discussion autour de l'uniformisation méthodologique; et plusieurs aspects de l'organisation des responsabilités de recherche et de décision en terminologie.

$\grave{A}$ notre avis on ne fait pas assez de différence entre la terminologie et les lexiques de spécialité; et entre les travaux réalisés pour la normalisation de la langue et ceux qui mènent à la normalisation des disciplines scientifiques et techniques. Ce fait produit une confusion des bases épistémologiques et méthodologiques de la terminologie. Il est possible qu'en des endroits ayant une situation linguistique conflictuelle, on traite la terminologie comme une affaire plutôt linguistique, ce qui peut donner de bons résultats si les scientifiques et les techniciens se sentent engagés dans le processus de normalisation, mais cela n'arrive pas toujours.

En deuxième lieu, il faut distinguer entre la normalisation de la terminologie des domaines de travail, la normalisation relative à l'usage terminologique des professionnels concernés, et la normalisation de la terminologie des disciplines scientifiques et techniques, qui inclut la normalisation conceptuelle de la discipline. Régler la terminologie d'une entreprise n'est pas la même chose qu'établir la terminologie d'une matière scientifico-technique.

En troisième lieu, il faudrait, à notre avis, éclaircir les différentes conceptions de la «terminologie», oublier la préminence linguistique en terminologie ST et stimuler le travail terminologique actif des spécialistes pour améliorer le processus de recherche.

Et enfin, nous pensons que les décisions sur l'organisation linguistique, la politique terminologigue et la distribution des tâches à faire ne peuvent pas se reproduire exactement d'un pays à un autre, même si elles ont produit des bons résultats en d'autres endroits. Seule une bonne connaissance de la situation spécifique d'une langue doit orienter les décisions organisatrices. Les situations peuvent se ressembler d'un pays à un autre, mais elles ne sont jamais les mêmes. 
NOTE:

1. Les paramètres de «spécialisation» sont le degré de connaissance des mots par le locuteur, le degré d'usage des mots en situations de la vie quotidienne ou en situations de communication spécialisée, l'usage conditionné sélectivement par l'interlocuteur, la présence dans le dictionnaire de langue, l'usage dans les médias, etc.

\section{BIBLIOGRAPHIE}

AUGER, P. (1980): «La normalisation terminologique et le rôle du spécialiste», Actes du troisième colloque OLF-STQ. Le rôle du spécialiste dans les travaux de terminologie, Québec, Office de la langue française.

CORBEIL, J-C. (1989): «Comment orienter l'usage d'une langue», La linguistique appliquée, Publicacions de la Universitat de Barcelona (sous presse).

FELBER, H. (1984): «Trends in terminology», Wien, Infoterm, 5-84.

GALINSKI, Ch, Budin, G. (1989): «El paper de l'especialista en terminologia», Séminaire réalisé à Barcelone en avril 1989.

GALINSKI, Ch, Nedobity, W. (1986): «Special Languages, Terminology Planning and Standardisation», Wien, Infoterm, $2-86$

GAUMOND, J-C. (1980) : «Le rôle des spécialistes dans les comités interentreprises de terminologie», Actes du troisième colloque OLF-STQ. Le rôle du spécialiste dans les travaux de terminologie. Québec, Office de la langue française.

ROUSSEAU, L-J. (1980): «Le rôle du spécialiste dans les travaux terminologiques systématiques», Actes du troisième colloque OLF-STQ. Le rôle du spécialiste dans les travaux de terminologie, Québec, Office de la langue française.

SAGER, J.C., DUNGWORTH, D., MCDONALD, P. (1980): English Special Languages, Wiesbaden, Brandstetter. 